\title{
Epithelial Cell Reconstruction and Visualization of the Developing Drosophila Wing Imaginal Disc
}

\author{
David E. Breen*\# \\ Drexel University \\ Philadelphia, PA, USA
}

\author{
Thomas Widmann ${ }^{\dagger} \#$ \\ Centro de Genómica e \\ Investigación Oncológica \\ Granada, Spain
}

\author{
Linge Bai \\ Drexel University \\ Philadelphia, PA, USA
}

\author{
Frank Jülicher ${ }^{\S}$ \\ Max Planck Institute for the \\ Physics of Complex Systems \\ Dresden, Germany
}

\author{
Christian Dahmann ${ }^{\text {II }}$ \\ Dresden University of Technology \\ Dresden, Germany
}

\begin{abstract}
Quantifying and visualizing the shape of developing biological tissues provide information about the morphogenetic processes in multicellular organisms. The size and shape of biological tissues depend on the number, size, shape, and arrangement of the constituting cells. To better understand the mechanisms that guide tissues into their final shape, it is important to investigate and measure the cellular arrangements within tissues. Here we present a set of techniques that produces detailed 3D models of the individual cells in an epithelial sheet. The inputs to the techniques are a volumetric model of an epithelium and a mesh model of the cell boundaries lying on its apical surface. The techniques include: definition of a Region of Interest (ROI), projection of the ROI vertices first to the basal surface then to the apical surface, projection of apical cell faces to the basal surface, creation of 3D epithelial cell models, and calculation and visualization of length and volume for each cell. In their first utilization we have applied these techniques to construct the individual epithelial cells of the wing imaginal disc of Drosophila melanogaster. To date, 3D epithelial cell models have been created, allowing for the calculation and visualization of cell parameters. The results show position-dependent patterns of cell shape in the wing imaginal disc. Our procedures should offer a general data processing pipeline for the construction of detailed 3D models of a wide variety of epithelial tissues.
\end{abstract}

Index Terms: I.3.5 [Computer Graphics]: Computational Geometry and Object Modeling-Geometric algorithms; I.3.8 [Computer Graphics]: Applications-Biomedical

\section{INTRODUCTION}

Biological tissues display a large diversity of shapes and sizes. The shape and size of tissues depend on the interplay between chemical signals (morphogens) and cell mechanics. This interaction also determines the shape of individual cells within the tissue. While chemical signals important for tissue development have been identified, relatively little is known about the mechanical properties of cells and tissues. Quantification and visualization of cell morphology and cell behavior provide a basis for understanding tissue mechanics and the role that cell shape plays in determining the overall structure of the tissue. The goal of the reported effort is to produce tools for the creation of detailed 3D geometric models of the individual cells in epithelial tissues. In our previous work [3], our tools

\footnotetext{
*e-mail: david@cs.drexel.edu, " Joint first authors

†e-mail: thomas.widmann@genyo.es

†e-mail:1b353@cs.drexel.edu

§e-mail: julicher@pks.mpg.de

Ile-mail: christian.dahmann@mailbox.tu-dresden.de
}

have been employed to create 3D volumetric surface models of the larval wing primordium of the fruit fly Drosophila melanogaster and to specify the apicolateral cell boundaries on the reconstructed surface.

The larval Drosophila wing primordium, the so-called pouch region of the wing imaginal disc, is an excellent model system to study epithelial morphogenesis during development. In this singlelayered main epithelium initially cuboidal cells elongate during development, forming a pseudostratified epithelium composed of highly columnar cells at later stages. Morphogen gradients (Dpp and Wingless), derived from two linear sources perpendicular to each other in the center of the wing disc, most likely control this cell shape transition $[19,20]$. To better understand the mechanisms that guide this transition, quantitative measurements of morphological parameters, such as cell area, cell height and cell volume, will be required.

In this paper, we describe a set of techniques that, when applied to a volumetric model of the fly's wing imaginal disc and a mesh model of it apicolateral cell boundaries, produces detailed 3D models of the individual cells in the epithelium. The techniques are based on a number of assumptions and utilize the geometric properties of the volumetric model to project the apicolateral cell mesh onto the basal surface of the wing disc; thus creating a full 3D description of the cells. Once the individual cells are defined, their length and volume may be computed and visualized, providing quantitative and visual data about the patterns of cell shapes and sizes in the wing disc epithelium. The 3D models which provide the evidence are produced via a data processing pipeline that is described in detail in Section 4. A number of reconstructed models of the epithelial cells of the Drosophila wing disc are presented to demonstrate the efficacy and promise of our approach.

\section{Related Work}

The work most related to ours is focused on reconstruction of the cellular structures of biological samples. A recent review on image analysis of embryo development [13] places our work into the larger context of imaging for developmental biology and references many of the papers described below.

Miodownik et al. [5, 17] create 3D models of an early-stage Drosophila melanogaster embryo with a mixture of imaging and interactive modeling techniques. The ellipsoidal shell that they create is used for finite element modeling of the ventral furrow invagination of the embryo in both $2 \mathrm{D}$ and $3 \mathrm{D}$. Müller-Reichert et al. [16] construct 3D models of individual neurons from the dorsal nerve cord of Caenorhabditis elegans based on scanning electron microscopy images. The images are manually segmented using the Imaris software (Bitplane, Zurich, Switzerland) [1], and the segments are formed into 3D models with the IMOD software $[2,10]$.

Mosaliganti et al. [15] describe a three-stage approach for reconstructing 3D cell nuclear structures from optical microscopy images of stained cell nuclei. The three stages include: a rough segmenta- 


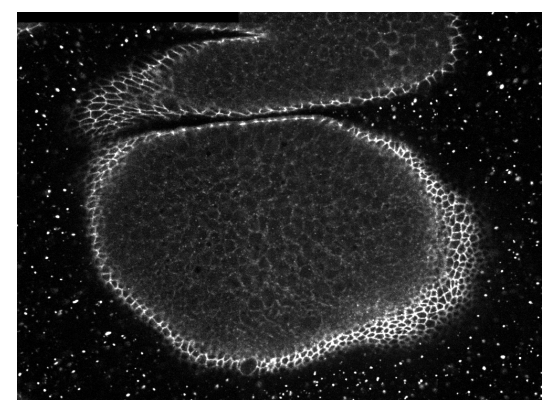

(a)

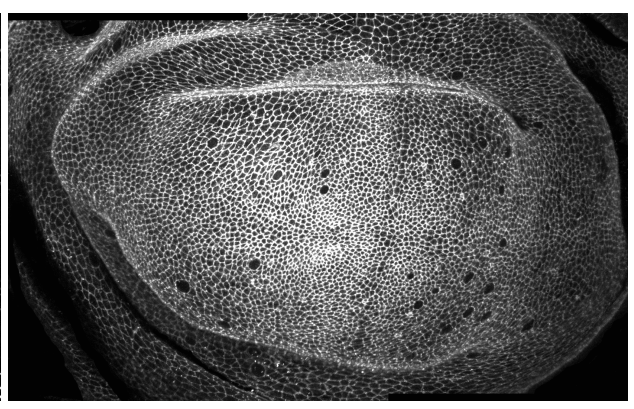

(b)

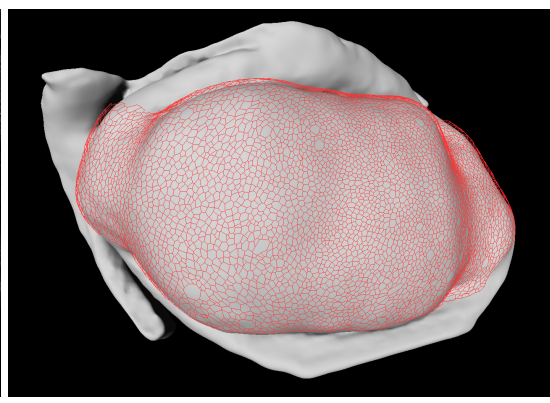

(c)

Figure 1: (a) Single blue-channel image from a confocal microscopy image stack of the wing imaginal disc of a larval Drosophila. The blue channel picks up an E-cadherin stain, a marker of adherens junctions, which is predominantly found on the apical side of the wing disc.

(b) Composite image displaying the apicolateral cell boundaries of the specimen. (c) 3D reconstruction of a wing disc with a 3D mesh representing the cell boundaries overlaid. Details of the reconstruction method can be found in [3].

tion of cell nuclei, refinement of the segmentation based on tessellations and slice interpolation. The major contribution of the paper is related to 2D segmentation of stained cell nuclei.

A 3D digital atlas of $C$. elegans has been built by staining and segmenting cell nuclei of newly hatched worms [12]. Based on stained nuclei detection algorithms applied to images obtained by digital scanned laser light sheet fluorescence microscopy, the dynamic 3D positions of the cell nuclei in a developing zebrafish embryo are computed and tracked [9] to produce a high resolution spatial/temporal dataset that captures numerous features of zebrafish embryogenesis. Digital scanned laser lightsheet fluorescence microscopy is then combined with incoherent structuredillumination microscopy to produce the 3D locations of stained nuclei in Drosophila embryos [8].

A contour grouping algorithm based on three geometric metrics is proposed by Leung et al. to determine the correspondences between cell outlines in adjacent images in an image stack [11]. This algorithm, which could be used during the $3 \mathrm{D}$ reconstruction of individual cells, achieved high accuracy when tested with synthetic data sets and with contours produced by manual segmentation of confocal images of gastrulating zebrafish cells.

4D reconstructions of the individual cells of a developing Arabidopsis thaliana flower are generated via 3D imaging, reconstruction and tracking of stained floral meristem membranes [6]. The technique utilizes manual landmark specification, automated registration and a 3D watershed algorithm to produce 3D models of the cells. Cell growth is tracked by computing a deformation field based on a manually identified surface, pairwise registration of sequential images and cell lineage updates.

Our work stands apart from previous work in that we have developed techniques to generate 3D models of individual epithelial cells from imaging data that only capture the tissue's apical and basal surfaces. This incomplete data is produced from imaging pseudostratified epithelia like the late-larval wing imaginal disc, because the epithelium's cells are too densely packed for its lateral sides to be traced from the apical to the basal surface. Our initial input data therefore capture cell features only on the apical and basal sides of the wing disc, with little imaging information present in the disc's interior, as seen in Figure 1(a). Therefore previously developed techniques would not be effective for our type of input images, because they either rely on whole tissue nuclear staining $[8,9,12,15]$ or are provided with interior membrane staining $[6,16]$ that is unavailable in our images.

\section{FOUNDATIONAL WORK}

In the earlier stage of our work techniques were developed to create $3 \mathrm{D}$ volumetric surface models of the wing imaginal disc, along with a geometric description of the epithelial cell boundaries on the apical side of the disc [3]. The input to this process is a stack of high resolution $(\sim 1024 \times 1024$ pixels $)$ confocal microscopy images of the wing discs. The specimens are immunostained for E-cadherin, a marker of the adherens junctions on the apical cell face boundaries, and stained with phalloidin, a marker of filamentous (F-) actin. Entire cell boundaries are highlighted by the F-actin meshwork underlying the cell plasma membrane. Figure 1(a) contains a single blue-channel image from an image stack that highlights the E-cadherin on the apical side of the wing disc. Each image in the stack is manually edited to isolate the apical surface. The edited images are merged using a maximum intensity projection to produce a single image that captures the apicolateral cell boundaries of the wing disc. See Figure 1(b). A 2D geometric description of the cell boundaries is then extracted from the image. The original stack is once again manually processed to produce contours that specify the basal and apical surface boundaries in each input image. These contours are the input to a $3 \mathrm{D}$ reconstruction process [4] that generates a 3D volumetric surface model of the entire wing disc using Multi-level Partition of Unity (MPU) implicit functions [18]. The $2 \mathrm{D}$ apical cell mesh is then projected onto the $3 \mathrm{D}$ model to generate the 3D locations of the cell boundaries on the apical surface. Figure 1(c) presents a reconstructed 3D model of a Drosophila wing imaginal disc with its 3D apical cell face mesh. Once the 3D mesh is generated it may be used to calculate and visualize apical cell face areas for a specimen.

\section{Epithelial Cell Reconstruction}

For each confocal image stack our previous work produces a $3 \mathrm{D}$ volumetric, implicit model of the wing disc specimen, along with a 3D polygon mesh that describes the epithelial cell face boundaries on the apical surface of the wing disc. This 3D volumetric model and 3D mesh are the input to the data processing pipeline for reconstructing the individual cells in an epithelium. The reconstruction method is based on the assumption that the centerline of an epithelial cell can be inferred by the surface normal at the basal side of the cell. This leads to a strategy of defining a region of interest on the basal surface of a sample, and projecting that region onto the apical surface following the basal-side surface normals. This strategy assumes that the epithelial cells extend in a straight line between the apical and basal surfaces of a specimen, an assumption that is not strictly true, but is not unreasonable for the purpose of calculating aggregate geometric cell parameters for a specimen. The data processing pipeline includes the following stages: definition of a Region of Interest (ROI), projection of the ROI vertices (in the $Z$ direction) first to the basal surface then (in the direction of the basal-side surface normals) to the apical surface, projection 


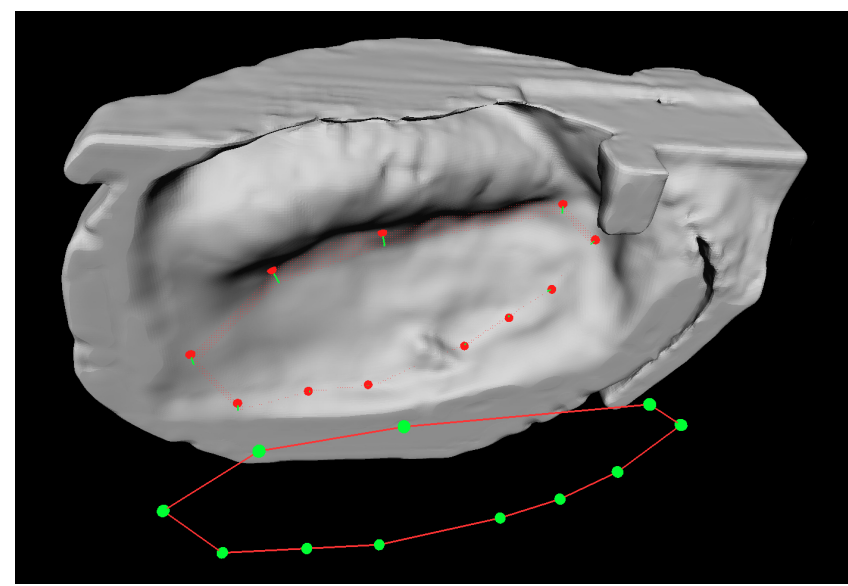

Figure 2: An ROI polygon (with green-colored vertices) positioned beneath the basal surface of a reconstructed wing disc model. The projected vertices lie on the basal surface and are colored red. Green lines represent the surface normals at the intersection points.

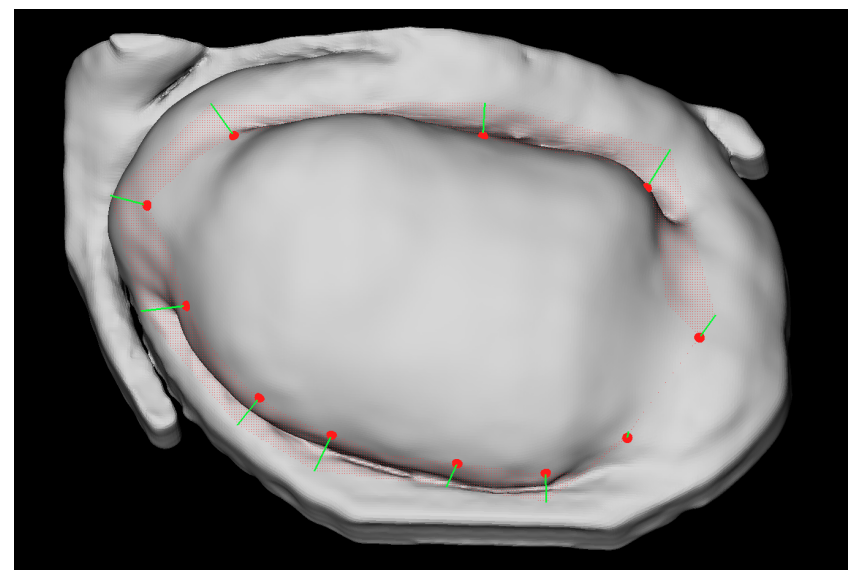

Figure 3: ROI vertices projected onto the apical surface of a reconstructed wing disc model. Green lines represent the projection direction.

of apical cell faces to the basal surface, creation of 3D epithelial cell models, and calculation of individual cell length and volume. Color-based visualization of these cell parameters may then be produced from the individual geometric cell models.

\subsection{Definition and Projection of ROI Vertices}

The first step of the process involves the definition of a Region of Interest (ROI) on the basal side of the reconstructed surface. The ROI is defined as a $2 \mathrm{D}$, convex polygon. The coordinate system of the 3D surface is aligned with the original input image stack, with the $X, Y$ coordinates corresponding to the $i, j$ pixel coordinates of the images, and the $Z$ coordinate corresponding to the image number in the stack. Appropriate scaling is applied in order to create a physically accurate 3D Cartesian space. Given this definition of the coordinate system, the 2D ROI polygon is specified by a user in the $X, Y$ plane. The $2 \mathrm{D}$ polygon is placed below the basal side of the $3 \mathrm{D}$ model. The volumetric model produced by MPU implicit functions has positive function values inside the model and negative values outside. The ROI polygon vertices can be projected onto model by incrementing the $Z$ component of each vertex and evaluating the model's implicit function value at the vertex's current 3D location. Detecting a sign change between increments indicates that the ver-

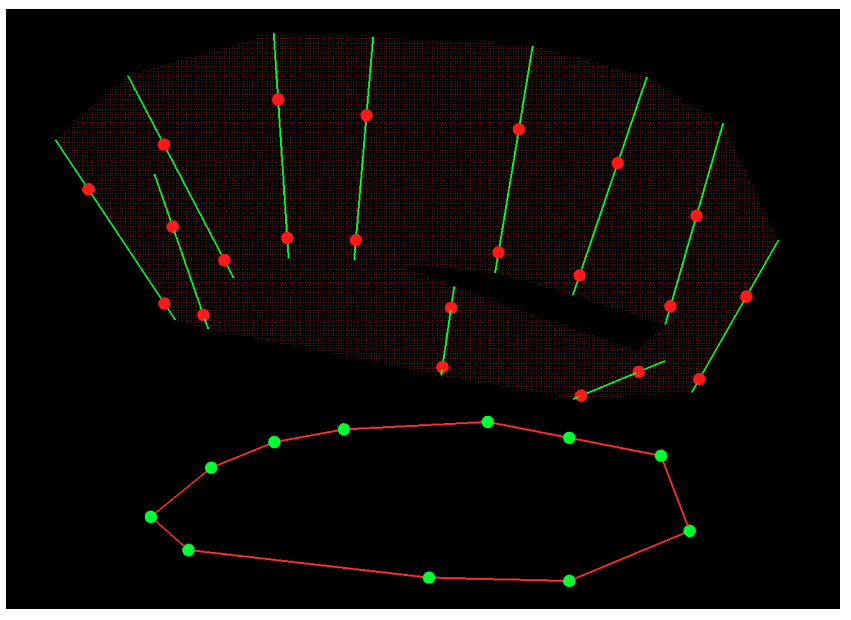

Figure 4: The original ROI polygon, with green vertices. The locations of the vertices after projection to the basal surface (lower red spheres), followed by a projection to the apical surface (upper red spheres). Green lines represent the projection direction.

tex has crossed the surface boundary. Linear interpolation is then used to calculate the exact intersection point. Figure 2 presents an ROI polygon positioned beneath the basal surface of the wing disc model. The original ROI vertices are colored green. These vertices have been projected in the $Z$ direction until they intersect with the surface. The projected ROI vertices lying on the surface are colored red. The surface normals at the intersection points are visualized as green lines.

Next, the vertices lying on the basal surface are projected in the direction opposite to surface normal until they intersect with the apical surface of the model. This direction vector is calculated at each basal-side vertex as the gradient of the underlying MPU implicit function using a finite difference scheme. The MPU field is stored as a discrete volume dataset, therefore MPU field values away from integer grid locations are calculated with tri-linear interpolation. As with the first projection calculation, the vertices are incrementally moved in the gradient direction until a sign change is detected in the MPU field. Linear interpolation between the two increment locations produces the exact intersection point on the apical surface. Figure 3 presents the projected ROI vertices lying on the apical surface of the reconstructed wing disc model. The green lines represent the projection direction, i.e. the negative surface normals at the vertices' basal locations. Figure 4 displays just the ROI polygon and the projected vertices. The green lines represent the projection direction from the basal to apical surface, with the lower red spheres lying on the basal surface and the upper spheres lying on the apical surface.

\subsection{Project Apical Cell Faces}

The ROI projection stage defines associated surface regions on the apical and basal surfaces of the wing disc model. We assume that the cells whose faces fall within the ROI region on the apical surface extend down into the ROI region on the basal surface. Given this assumption a purely geometric computation may be used to project apical cell faces onto the basal surface. Since the 3D apical cell face mesh extends beyond the apical ROI region, the cell faces lying inside the apical ROI region must first be identified. The 3D cell face mesh is created by projecting a $2 \mathrm{D}$ cell mesh in the $Z$ direction [3]. In other words the $X, Y$ locations of the cell face vertices do not change during this projection step, ensuring that no cell faces overlap or change their spatial ordering. Therefore a $2 \mathrm{D}$ point-inpolygon test [7] may be utilized. Every vertex of every 3D cell 


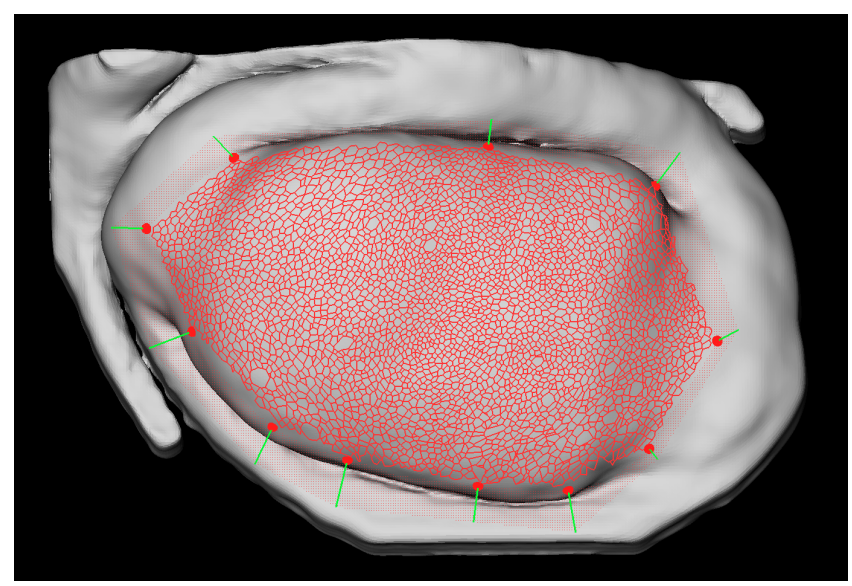

Figure 5: A subset of the 3D cell face mesh is identified as inside of the apical ROI polygon. Note that some of the cell faces displayed in Figure 1(c), those outside of the apical ROI, have been trimmed away. .

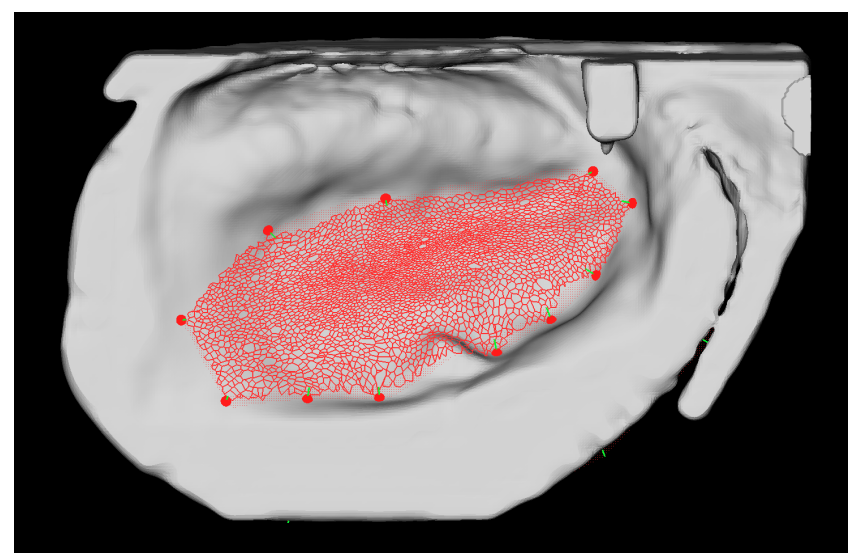

Figure 6: The trimmed apical cell face mesh is projected to the basal surface with projection directions calculated as a weighted sum of the surface normals at the basal ROI polygon vertices.

face is tested for inclusion inside the apical ROI polygon. Only the $X, Y$ coordinates of the cell and polygon vertices are utilized for this test, making it a 2D calculation. A cell face is considered inside the apical ROI polygon only if all of its vertices are inside of the polygon. Figure 5 displays the subset of the 3D cell face mesh that lies within the apical ROI polygon. Note that some of the cell faces displayed in Figure 1(c), those outside of the apical ROI, have been trimmed away.

The apical cell faces inside the apical ROI polygon are then projected down onto the basal surface. The general strategy for this projection step involves calculating a unique projection direction for each vertex of the trimmed apical cell face mesh. The projection direction $D_{i}$ for vertex $V_{i}$ is calculated as a weighted sum of the projection directions $R_{j}$ used to project the basal ROI polygon vertices $B_{j}$ to the apical surface, producing the apical ROI vertices $P_{j}$. The $R_{j}$ directions are visualized as green lines in Figure 4. The $P_{j}$ vertices are the upper red spheres. More succinctly put

$$
D_{i}=\sum_{j=1}^{J} \alpha_{j}^{i} R_{j},
$$

where $J$ is the total number of vertices in an ROI polygon and the weights $\alpha_{j}^{i}$ are the barycentric coordinates of $V_{i}$ relative to the apical

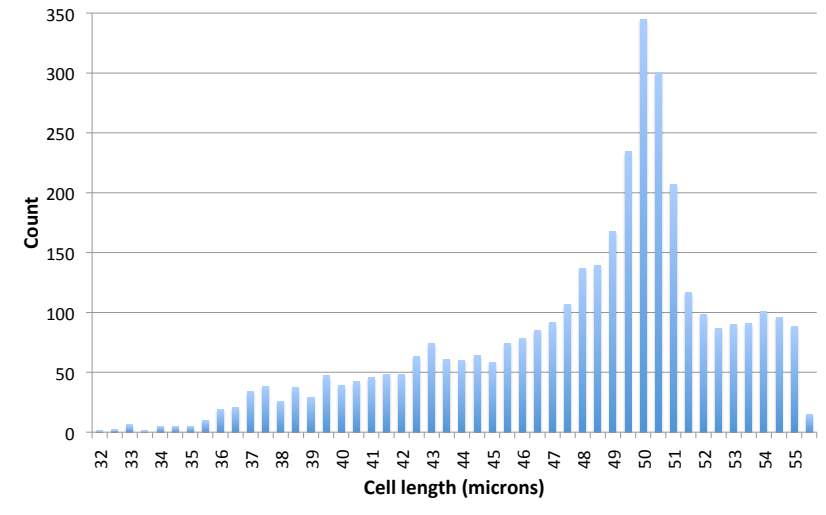

Figure 7: Histogram of cell lengths calculated for the model presented in Figures 5, 6 and 11.

ROI vertices $P_{j}$. The weights $\alpha_{j}^{i}$ are calculated using the method described by Meyer et al. [14] applied to only the $X, Y$ components of the $V_{i}$ and $P_{j}$ vertices. A minor constraint of the method is that the ROI polygon must be convex in order to produce valid results.

Each apical cell face vertex $V_{i}$ is projected in the negative $D_{i}$ direction until it crosses the basal surface. As before, the position of $V_{i}$ is incremented in the $D_{i}$ direction until a sign change is detected in the MPU scalar field. Linear interpolation moves the projected vertex to the zero-crossing of the implicit model, leaving it on the basal surface. Figure 6 presents the cell face mesh produced by projecting the trimmed apical cell face mesh to the basal surface. Note that the cell faces have become constricted because of the curved structure of the wing disc.

\subsection{Creation and Evaluation of 3D Epithelial Cell Models}

After the apical-to-basal projection step, there is sufficient information for defining a 3D cell model for each cell lying inside the ROI polyhedron, which is defined by the apical ROI polygon and the basal ROI polygon. A 3D epithelial cell model is defined by a number of parameters. They are:

- Cell Face - Ordered set of 3D vertices, i.e. a 3D polygon. Each cell has two faces, one on the apical surface and another on the basal surface of the wing disc model.

- Cell Face Center - Average location of a cell face's vertices. A center is calculated for both apical and basal cell faces.

- Cell Centerline - Vector pointing from the basal cell face center to the apical cell face center.

- Cell Length $L_{i}$ - The length of a cell's centerline, i.e. the distance between the apical cell face center and the basal cell face center.

Additionally, the volume of the cell can be calculated from these parameters. For each 3D cell face a 2D projected cell face is calculated. The 3D vertices of a cell face are projected into the plane defined by the cell face center and the cell centerline, making the projected cell face coplanar and perpendicular to the centerline. The area of the projected cell face $A_{\text {proj }}$ is calculated as the sum of the areas of the triangles produced by connecting the cell face center to pairs of vertices of the projected cell face. The area of the projected, coplanar cell face is calculated instead of the area of the tilted (with respect to the cell's centerline), non-coplanar, possibly distorted face in order to produce a more accurate cell volume $V_{o l}$ calculations, which is defined as

$$
\operatorname{Vol}_{i}=\left(A_{\text {proj }}^{\text {apical }}+A_{\text {proj }}^{\text {basal }}\right) / 2 * L_{i},
$$




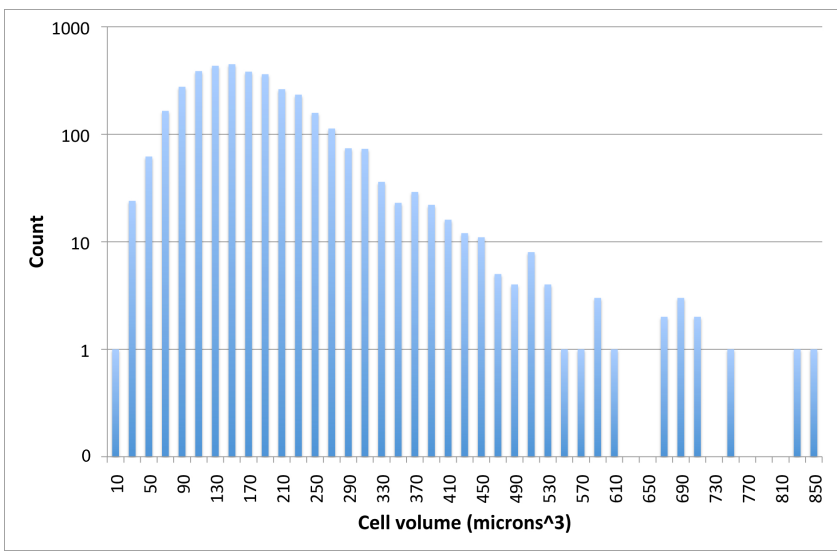

Figure 8: Histogram of cell volumes calculated for the model presented in Figures 5, 6 and 12. Note that the cell counts are given with a logarithmic scale.

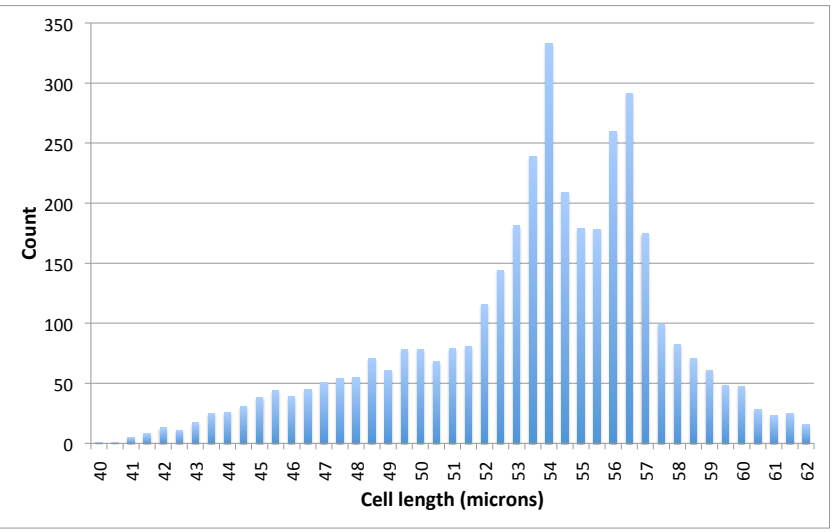

Figure 9: Histogram of cell lengths calculated for the model presented in Figure 13.

which is the average of the two projected cell face areas times the length of the cell.

Figure 7 presents a histogram of the individual cell lengths computed from the wing disc model of Figures 5 and 6 . Note that most cells are approximately 50 microns in length. Figure 8 presents a histogram of the individual cell volumes computed from the same model. Figures 9 and 10 contain histograms of the cell lengths and volumes for the reconstructed model presented in Figures 13 and 14. The cell lengths in this wing disc show a bimodal distribution with peaks at 53.5 and 56.5 microns. Further study is needed to determine if such a bimodal distribution is common in Drosophila wing discs.

Note that the histogram counts in Figures 8 and 10 are plotted with a logarithmic scale, since a large majority of the cell volumes are in the range 70 to 280 microns $^{3}$. Plotting this data with a linear scale would make interpreting the cell counts outside this range difficult. It should also be noted that the cell volume histograms do not include a small number (3) of large, dividing cells that have a volume greater than 1200 microns $^{3}$, since including these cells would create histograms with a large swath of zero entries. These excluded cells are dividing and their nuclei have migrated to the apical surface, greatly exaggerating their apical cross-sections. It can also be seen that the histograms of Figures 8 and 10 contain a small number of large-volume cells (volumes greater than 550 microns $^{3}$ ). The nuclei of these cells are also lying directly beneath the apical surface, which enlarges the cells' apical faces. These exaggerations

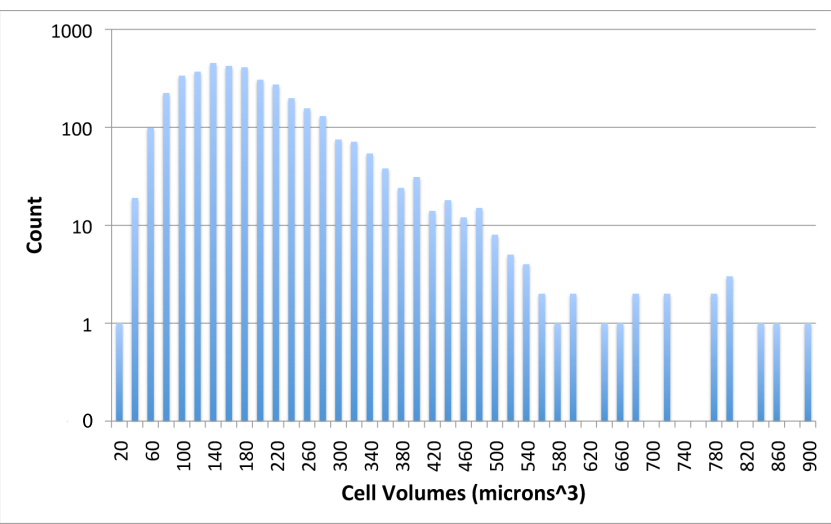

Figure 10: Histogram of cell volumes calculated for the model presented in Figure 14. Note that the cell counts are given with a logarithmic scale.

lead to an overestimation of individual cell volumes, a fact that is not significant when calculating gross, averaged geometric parameters, since the specimens mostly contain non-dividing cells whose nuclei are not near the surface.

\section{Visualization of Epithelial Cell Parameters}

For visualization purposes the calculated lengths and volumes are mapped to the Hue channel of the HSV color space. The linear mapping

$$
H=((\text { max_length }- \text { length }) /(\text { max_length }- \text { min_length })) * 240
$$

is used to visualize cell lengths and produces a color that is applied to the cells' faces. The quadratic mapping

$H=\left(\left(\max \_ \text {volume }- \text { volume }\right) /\left(\max \_ \text {volume }-\min \_ \text {volume }\right)\right)^{2} * 240$

is utilized for visualizing cell volume. For these mappings, the resulting color distribution ranges from dark blue for the smallest cells to bright red for the largest. The quotient is squared in the volume-to-hue mapping in order to produce a greater color spread in the blue to green range, which provides better differentiation of the cells in the center of the disc. Since a few large, dividing cells skew the color map in the yellow to red range, changing the color mapping from linear to quadratic improves the color distribution in the most populated range of cell face volumes ( 70 to 280 microns $^{3}$ ).

\section{Results and Discussion}

Figures $11,12,13$ and 14 present the results of applying our reconstruction and visualization techniques to two Drosophila wing imaginal disc models. The model in Figures 11 and 12 was reconstructed from a stack of 76 confocal images, with a resolution of $1669 \times 1044$, of a Drosophila wing imaginal disc at 108 hours AEL (after egg lay). The surface reconstruction process (described in [3]) produced a volumetric representation of the wing disc with a resolution of $838 \times 526 \times 310$ and identified 4,951 cell faces on its apical surface. The trimming process described in Section 4.2 produces 3,640 cell faces which are projected to the basal surface and subsequently define 3D epithelial cell models. Once these models were defined their length and volume were computed and visualized. See Figures 7, 8, 11 and 12. The geometric parameter values computed for the model are similar to the estimates derived directly from the images and correlate with the values generally accepted within the biological community for these quantities. The last two figures contain color keys that visually describe the mapping from 

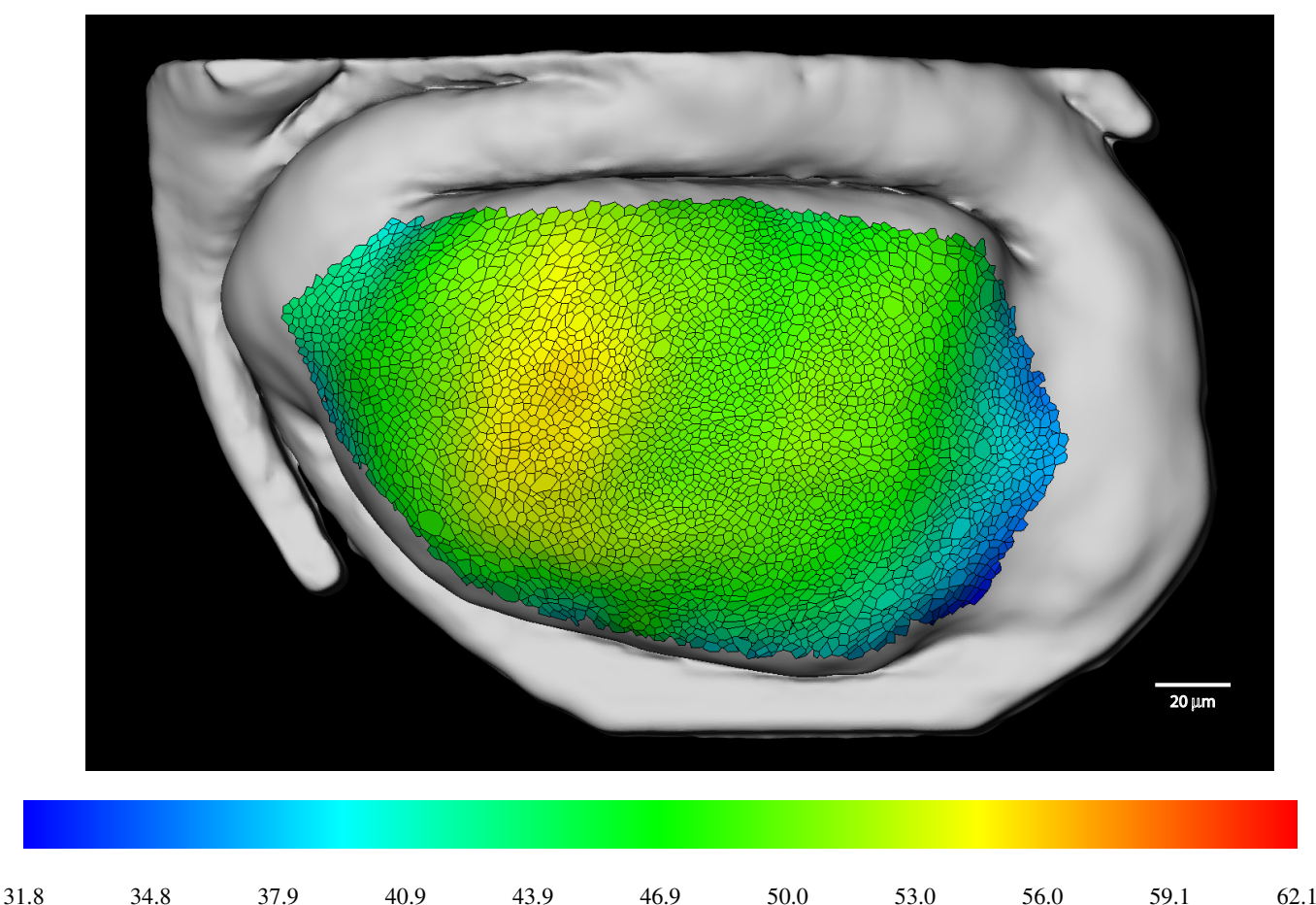

Figure 11: Reconstructed Drosophila wing imaginal disc at 108 hours AEL (after egg lay). The cell faces have been color shaded as a function of the length of the cell. Lengths in the color key are given in microns.
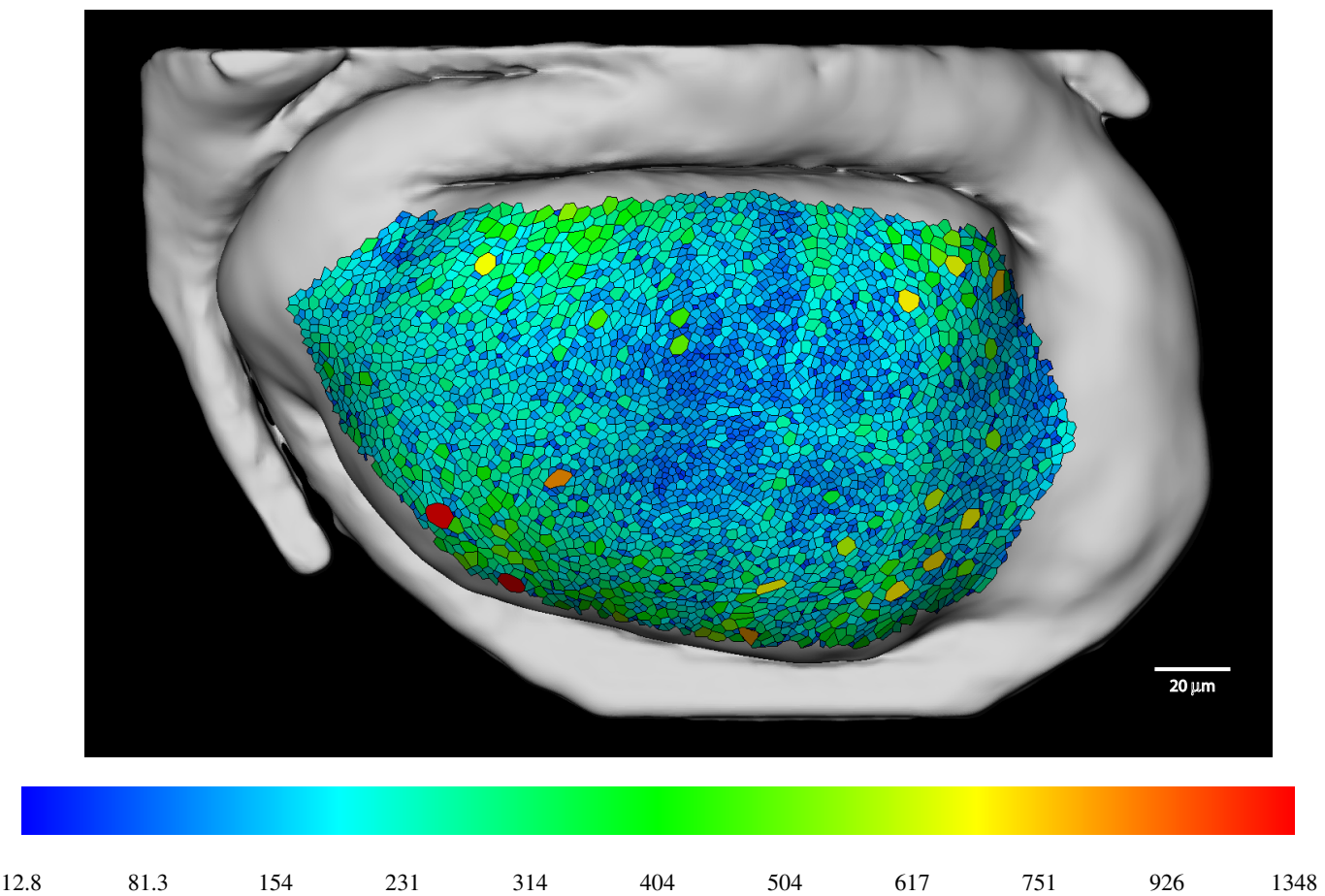

Figure 12: Reconstructed Drosophila wing imaginal disc at 108 hours AEL. The cell faces have been color shaded as a function of the cell's volume. Volumes in the color key are given in microns ${ }^{3}$.

cell length and volume to color. Note that these keys span the minimum and maximum values computed from the two example models, to allow for the consistent visual comparison of the models.

The model in Figures 13 and 14 was reconstructed from a stack of 43 confocal images, with a resolution of $1941 \times 1125$, of another wing disc at 108 hours AEL. A volumetric model with resolution $946 \times 578 \times 283$ was produced from these images and 5,635 cell faces were identified on the wing disc's apical surface. The trim- 


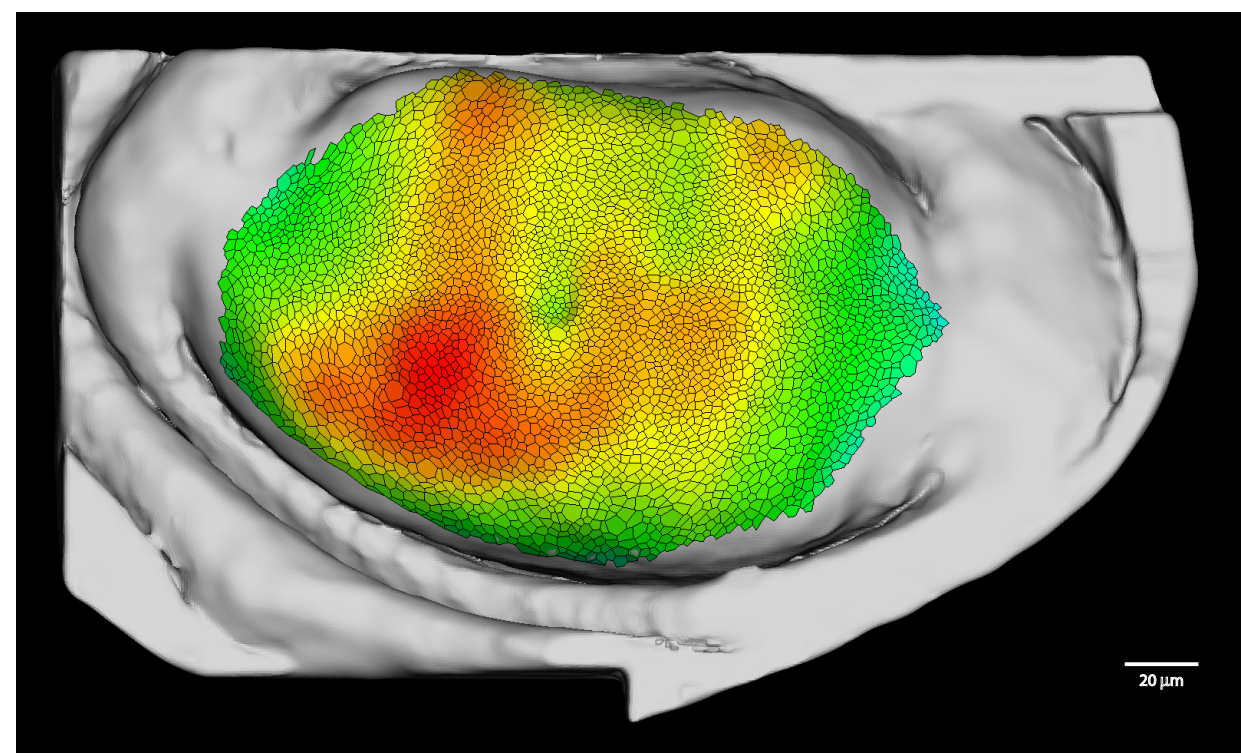

Figure 13: Another reconstructed Drosophila wing imaginal disc at 108 hours AEL (after egg lay). The cell faces have been color shaded as a function of the length of the cell. Lengths in the color key are given in microns.
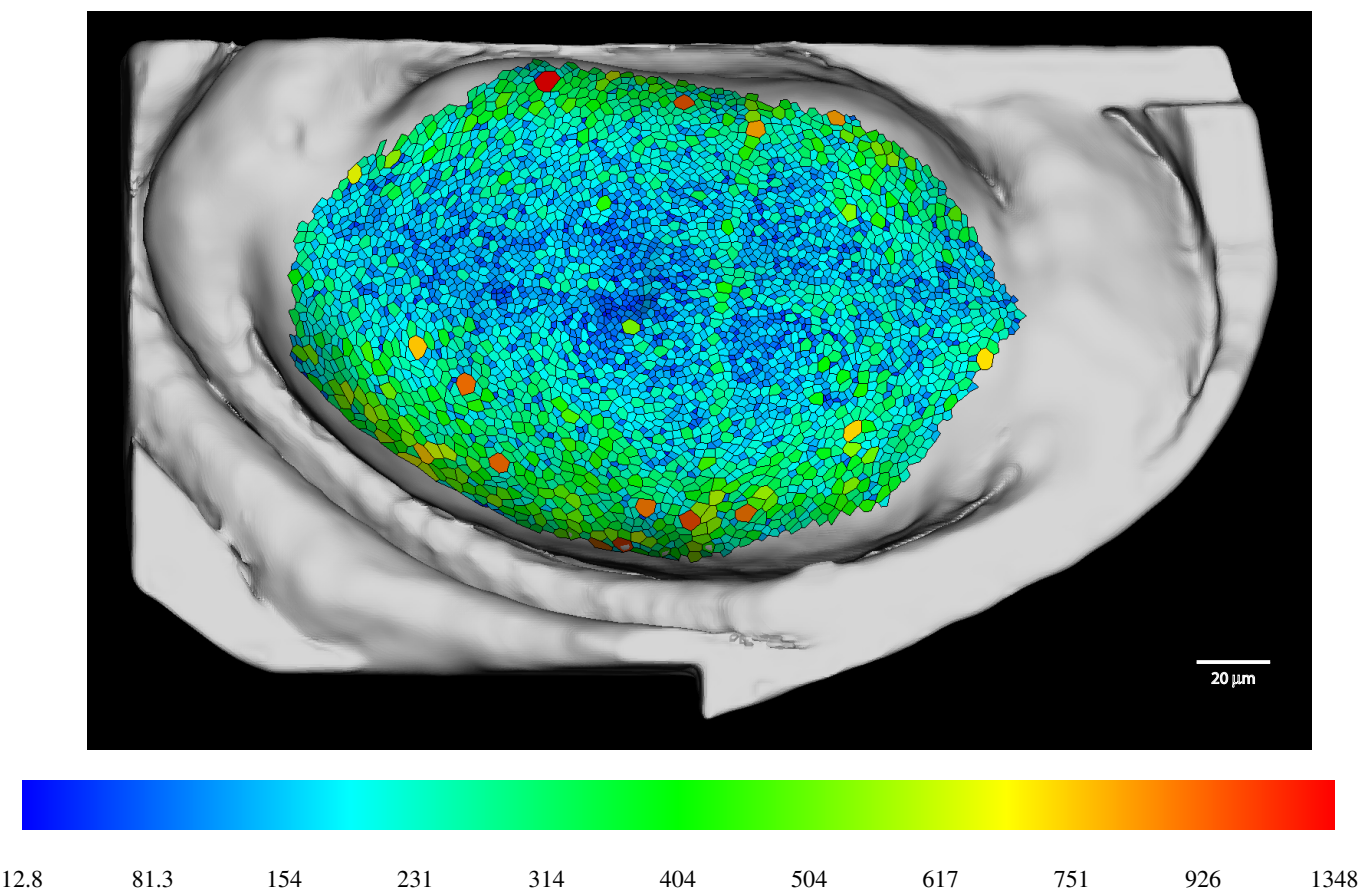

Figure 14: Another reconstructed Drosophila wing imaginal disc at 108 hours AEL. The cell faces have been color shaded as a function of the cell's volume. Volumes in the color key are given in microns ${ }^{3}$.

ming, projection and $3 \mathrm{D}$ cell generation process produced 3,785 3D epithelial cell models, whose geometric parameters were computed and visualized. The total computation time needed to complete all of the steps for generating and evaluating the 3D epithelial cell models is approximately 1 minute for each model on a 2.66 $\mathrm{GHz}$ dual-core MacBook Pro with 4 GB of memory.
The reconstructions and visualizations are useful to biologists because they indicate that cells have different lengths and volumes in the wing disc, depending on their location. Cells in a broad central region of the wing disc pouch are, on average, longer compared to cells in the periphery of the wing disc pouch. Cells in a narrow central region, in close proximity to the sources of the morphogens 


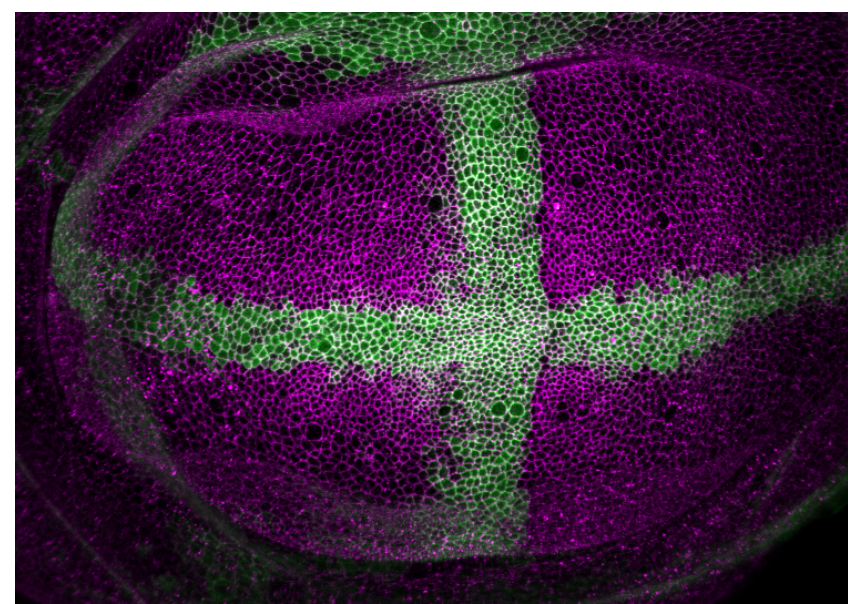

Figure 15: An apical surface of a wing imaginal disc at 108h AEL expressing membrane-bound CD8-GFP (green) under control of ptcGAL4 and wg-GAL4 drivers stained for E-cadherin (magenta). The vertical and horizontal stripes of CD8-GFP-expressing cells illustrate the sources of the Dpp and Wingless morphogens, respectively.

Dpp and Wingless, as seen is Figure 15, have smaller volumes compared to other cells. The stereotyped pattern of cell shapes observed in the wing imaginal discs visualizations might result from the activities of the Dpp and Wingless morphogens [19, 20]. This visual evidence encourages us to conduct future experiments that will determine the precise correlation between Dpp/Wingless morphogen gradients and patterns of cell shapes/sizes.

The quantitative data on cell shape that we are now able to compute provide an important basis for further experimental analysis and theoretical modeling of wing disc morphogenesis. For example, once several wing discs from different developmental stages are analyzed, it will be possible to compare the cell morphological parameters between them. This will provide us with i.) a quantitative description of the changes in wing disc cell morphology during development and ii.) mechanistic insight of the process (e.g. is cell volume conserved during the morphogenetic changes?)

\section{Conclusion}

We have described a set of techniques that may be used to produce detailed 3D models of the individual cells in biological epithelial tissues. The inputs to the techniques are a 3D volumetric model of the tissue and a mesh model of the cell faces lying on its apical surface. We have applied the techniques to the analysis of the developing imaginal wing disc of a late-larval Drosophila melanogaster. The 3D models are produced with a data processing pipeline that include: definition of a Region of Interest (ROI), projection of the ROI vertices first to the basal surface then to the apical surface, projection of apical cell faces to the basal surface, creation of 3D epithelial cell models, and calculation and visualization of individual cell length and volume.

Future work will apply these techniques to significant numbers of specimens from different developmental stages. The quantitative data on cell shape and cell topology that can be derived over developmental time spans from the 3D models will provide the groundwork for future biomechanical models of tissue morphogenesis.

\section{ACKNOWLEDGEMENTS}

We would like to thank Yutaka Ohtake for his MPU implicits software and Mark Meyer for his barycentric coordinates software. This research was funded by National Science Foundation grants ACI-0083287 and IIS-084541 (DB), a Drexel Synergy Grant (DB),
German Research Foundation grant DA586/8-1 (CD), and Max Planck Institute Visiting Scientist Fellowships (DB and LB).

\section{REFERENCES}

[1] Imaris software. http://www.bitplane.com/go/ products/imaris, Retrieved December 2, 2011.

[2] Imod software. http://bio3d.colorado.edu/imod/, Retrieved December 2, 2011

[3] L. Bai, T. Widmann, F. Jülicher, C. Dahmann, and D. Breen. 3D surface reconstruction and visualization of the Drosophila wing imaginal disc at cellular resolution. Technical Report DU-CS-12-05, Department of Computer Science, Drexel University, 2012.

[4] I. Braude, J. Marker, K. Museth, J. Nissanov, and D. Breen. Contourbased surface reconstruction using MPU implicit models. Graphical Models, 69(2):139-157, 2007.

[5] V. Conte, J. Muñoz, and M. Miodownik. A 3D finite element model of ventral furrow invagination in the Drosophila melanogaster embryo. Journal of the Mechanical Behavior of Biomedical Materials, 1(2):188-198, 2008.

[6] R. Fernandez, P. Das, V. Mirabet, E. Moscardi, J. Traas, J. Verdeil, G. Malandain, and C. Godin. Imaging plant growth in 4D: robust tissue reconstruction and lineaging at cell resolution. Nature Methods, 7(7):547-553, 2010.

[7] E. Haines. Point in polygon strategies. In P. Heckbert, editor, Graphics Gems IV, pages 34-45. Academic Press, Boston, 1994

[8] P. Keller, A. Schmidt, A. Santella, K. Khairy, Z. Bao, J. Wittbrodt, and E. Stelzer. Fast, high-contrast imaging of animal development with scanned light sheet-based structured-illumination microscopy. Nature Methods, 7(8):637-642, 2010.

[9] P. Keller, A. Schmidt, J. Wittbrodt, and E. Stelzer. Reconstruction of zebrafish early embryonic development by scanned light sheet microscopy. Science, 322(5904):1065, 2008.

[10] J. Kremer, D. Mastronarde, and J. McIntosh. Computer visualization of three-dimensional image data using IMOD. Journal of Structural Biology, 116:71-76, 1996

[11] T. Leung, J. Veldhuis, S. Krens, C. Heisenberg, and G. Brodland. Identifying same-cell contours in image stacks: A key step in making 3D reconstructions. Annals of Biomedical Engineering, 39:698-705, 2011.

[12] F. Long, H. Peng, X. Liu, S. Kim, and E. Myers. A 3D digital atlas of C. elegans and its application to single-cell analyses. Nature Methods, 6(9):667-672, 2009.

[13] M. Luengo-Oroz, M. Ledesma-Carbayo, N. Peyriéras, and A. Santos. Image analysis for understanding embryo development: a bridge from microscopy to biological insights. Current Opinion in Genetics \& Development, 2011.

[14] M. Meyer, H. Lee, A. Barr, and M. Desbrun. Generalized barycentric coordinates on irregular polygons. Journal of Graphics, GPU, and Game Tools, 7(1):13-22, 2002.

[15] K. Mosaliganti, L. Cooper, R. Sharp, R. Machiraju, G. Leone, K. Huang, and J. Saltz. Reconstruction of cellular biological structures from optical microscopy data. IEEE Transactions on Visualization and Computer Graphics, 14(4):863-876, 2008

[16] T. Müller-Reichert, J. Mancuso, B. Lich, and K. McDonald. Threedimensional reconstruction methods for Caenorhabditis elegans ultrastructure. Methods in Cell Biology, 96:331-361, 2010.

[17] J. Muñoz, K. Barrett, and M. Miodownik. A deformation gradient decomposition method for the analysis of the mechanics of morphogenesis. Journal of Biomechanics, 40(6):1372-1380, 2007.

[18] Y. Ohtake, A. Belyaev, M. Alexa, G. Turk, and H. Seidel. Multi-level partition of unity implicits. ACM Transactions on Graphics (Proc. SIGGRAPH), 22(3):463-470, 2003.

[19] T. Widmann and C. Dahmann. Dpp signaling promotes the cuboidalto-columnar shape transition of Drosophila wing disc epithelia by regulating Rho1. Journal of Cell Science, 122:1362-1373, 2009.

[20] T. Widmann and C. Dahmann. Wingless signaling and the control of cell shape in Drosophila wing imaginal discs. Developmental Biology, 344:161-173, 2009 